EUROPEAN JOURNAL OF PURE AND APPLIED MATHEMATICS

Vol. 12, No. 2, 2019, 448-468

ISSN 1307-5543 - www.ejpam.com

Published by New York Business Global

\title{
Existence and Uniqueness Solution under Non-lipschitz condition of the mixed fractional Heston's model
}

\author{
Didier Alain Njamen Njomen ${ }^{1, *}$, Eric Djeutcha ${ }^{1}$, Louis-Aimé Fono ${ }^{2}$ \\ 1 Department of Mathematics and Computer's Science, Faculty of Science, University of \\ Maroua, Maroua, Cameroon \\ 2 Department of Mathematics and Computer's Science, Faculty of Science, \\ University of Douala, Cameroon
}

\begin{abstract}
This paper focuses on a mixed fractional version of Heston model in which the volatility Brownian and price Brownian are replaced by mixed fractional Brownian motion with the Hurst parameter $H \in\left(\frac{3}{4}, 1\right)$ so that the model exhibits the long range dependence. The existence and uniqueness of solution of mixed fractional Heston model is established under various non-Lipschitz condition and a related Euler discretization method is discussed. An example on the American put option price using Least Squares Monte Carlo Algorithm to produce acceptable results under the mixed fractional Heston model is presented to illustrate the applicability of the theory. The numerical result obtained proves the performance of our results.
\end{abstract}

2010 Mathematics Subject Classifications: 60J65, 60G22, 60H07, 91B25, 91B26

Key Words and Phrases: Brownian motion, fractional processes, mixed fractional Brownian, Heston mode, Monte Carlo Algorithm

\section{Introduction}

The fractional Brownian motion (fBm) $B^{H}=\left\{B_{t}^{H}, t \in[0, T]\right\}$ with Hurst parameter $H \in(0,1)$ is a Gaussian self-similar process with stationary increments. This process was introduced by [18] and studied by [23], where a stochastic integral representation in terms of a standard Brownian motion (Bm for short) was established. The parameter $H$ is called Hurst index from the statistical analysis, developed by the climatologist [17]. The self-similarity and stationary increments properties make the $\mathrm{fBm}$ an appropriate model for many applications in diverse fields from biology to finance [19], [22]. If $H \neq \frac{1}{2}$, the process $B_{t}^{H}$ is neither a semimartingale, nor Markovian process. Therefore, the classical Ito calculus can not be used to analyze the fBm process. In order to overcome this problem, we use a mixed fractional Brownian motion (mfBm for short) with $a, b$ and $H$ parameters

${ }^{*}$ Corresponding author.

DOI: https://doi.org/10.29020/nybg.ejpam.v12i2.3395

Email addresses: didiernjamen1@gmail.com (D. A. N. Njamen), djeutchaeric@yahoo.fr (E. Djeutcha), Ifono2000@yahoo.fr (L-A. Fono) 
which is a linear combination of Brownian motion and fractional Brownian motion with parameter $H$, defined for any $t \in[0, T]$ by:

$$
M_{t}^{H}=\left\{a B_{t}+b B_{t}^{H}, \forall a, b>0\right\},
$$

where $B^{H}$ is the $\mathrm{fBm}$ with Hurst parameter $H \in(0,1)$. [10] proved that the $\mathrm{mfBm}$ process with Hurst parameter $H \in] \frac{3}{4}, 1$ [ is equivalent to a martingale $a B_{t}$ and hence it is arbitrage-free.

Let $T>0$ be a fixed time and $\left(\Omega, F,\left(F_{t}\right)_{t \in[0, T]}, \mathbb{P}\right)$ be a given filtered complete probability space with $\left(F_{t}\right)_{t \in[0, T]}$ being a filtration that satisfies the usual hypothesis.

The aim of this paper is to study the following stochastic differential equation (SDE for short) on $\mathbb{R}^{n}$

$$
d X(t)=\mu(t, X(t)) d t+\sigma(t, X(t)) d M_{t}^{H} .
$$

On most occasions, the coefficients of SDEs driven by mfBm are assumed to satisfy the Lipschitz condition. The existence and uniqueness of solutions of SDEs driven by fBm with Lipschitz condition have been studied in [13], [11]. Fortunately, many researchers have investigated the SDEs under non-Lipschitz condition and they presented many meaningful results [31], [5], [29, 30]. But, to the best of our knowledge, the existence and uniqueness of solutions of SDEs driven by $\mathrm{mfBm}$ with a non-Lipschitz condition have not been considered. This point motivates us to carry out the present study. In the present paper, we discuss the SDEs with mfBm under the non-Lipschitz condition. Using the successive approximation method, the existence and uniqueness theorems of solutions to the following non-Lipschitz SDEs driven by mfBm are proved:

$$
X(t)=x_{0}+\int_{0}^{t} \mu(s, X(s)) d s+\int_{0}^{t} \sigma_{1}(s, X(s)) d B_{s}+\int_{0}^{t} \sigma_{2}(s, X(s)) d B_{s}^{H},
$$

where $t \in[0, T], x_{0}=\xi \in \mathbb{R}^{n}$ is a random variable, $0 \leq T<\infty$, the process $B$ represent a $m$-dimensional standard $F_{t}$-Brownian motion and the process $B^{H}$ represent a $d$-dimensional $F_{t}$-adapted fractional Brownian motion with the Hurst index $H \in\left(\frac{3}{4}, 1\right)$ defined in a same complete probability space $(\Omega, \mathcal{F}, \mathbb{P})$, and $\mu(t, X(t))[0, T] \times \mathbb{R} \rightarrow \mathbb{R}, \sigma_{1}(t, X(t)):[0, T] \times \mathbb{R} \rightarrow \mathbb{R}$ and $\sigma_{2}(t, X(t)):[0, T] \times \mathbb{R} \rightarrow \mathbb{R}$ are all mesurable functions.

The main difficulty when considering Equation (3) lies in the fact that both stochastic integrals are dealt in different ways. However, the integral with respect to the $\mathrm{Bm}$ is an It integral, while the integral with respect to the $\mathrm{fBm}$ has to be understood in the pathwise sense. Finally, using the LSM Algorithm, we will calculate the value of the American put option price under the Heston model governed by $M^{H}$ ( mixed fractional Heston model (in short MFH)) for differents values of the Hurst parameter $H$ and we compare the result with the value of American option price under Heston Model (HM). We remind that in (3), $\int_{0}^{t} \cdot d B_{s}$ stand for the stochastic integral w.r.t Bm, $\int_{0}^{t} \cdot d B_{s}^{H}$ stand for the stochastic integral w.r.t $\mathrm{fBm}$.

The article is organized as follows. In section 2 , we recall briefly the malliavin calculus in order to define the integral with respect to $\mathrm{fBm}$ and introduce proper normed spaces and we also state our assumptions on the coefficients $\mu, \sigma_{1}$ and $\sigma_{2}$ of Equation (3).

In section 3, we give a version of Heston's model which the volatility Brownian and stock price Brownian are replaced by the mixed fractional Brownian motion. The main existence and uniqueness results are discussed under the non-Lipschitz condition and simulation result using the 
discritization of Euler's method for 80 simulation paths of the stock price is provided. Finally, in section 4, we give the applicability of the general theory to calculate the value of American put option price under the MFH model and we compare the result with the value of American put option price under the Heston Model (HM).

\section{Preliminaries}

The (elementary) background needed here about the theory of integral with respect fBm [26], $[20]$. Let $(\Omega, \mathcal{F}, \mathbb{P})$ be a complete filtered probability space satisfying the usual assumptions. We fix some notation throughout the paper $|\cdot|$ will denote the absolute value of a real number, the Euclidean norm of a vector, or the operator norm of a matrix. The symbol $K$ will denote a generic constant, whose value may change from one value to another. $\diamond$ denote the Wick product and is defined in [7]. If $A$ is a vector or matrix, its transpose is denoted by $A^{T}$.

Stochastic differential equation with respect to $\mathrm{fBm}$ have been interpreted via various stochastic integral, such as the Wick-integral, the Wiener integral, the Skorohod integral, and path-wise integral [26], [14], [2, 3], [9], [25]. In this paper, we consider the path-wise integral with respect to $\mathrm{fBm}[26]$.

\subsection{Stochastic integral with respect to $\mathrm{fBm}$.}

We begin by a brief review of the malliavin calculus. We start with the definition of the integral with respect to fBm as a path-wise stochastic integrals (symmetric, forward and backward integral) for $\mathrm{fBm}$ on the slow-fast systems, following the work of [7].

Definition 1. Let $u(t)$ be a stochastic process with integrable trajectories.

(i) The symmetric integral of $u(t)$ with respect to $B_{t}^{H}$ is defined as

$$
\lim _{\varepsilon \rightarrow 0} \frac{1}{2 \varepsilon} \int_{0}^{T} u(s)\left[B_{s+\varepsilon}^{H}-B_{s-\varepsilon}^{H}\right] d s
$$

provided that the limit exists in probability, and is denoted by $\int_{0}^{T} u(s) d^{\circ} B_{s}^{H}$.

(ii) The forward integral of $u(t)$ with respect to $B_{t}^{H}$ is defined as

$$
\lim _{\varepsilon \rightarrow 0} \frac{1}{\varepsilon} \int_{0}^{T} u(s)\left[\frac{B_{s+\varepsilon}^{H}-B_{s}^{H}}{\varepsilon}\right] d s,
$$

provided that the limit exists in probability, and is denoted by $\int_{0}^{T} u(s) d^{-} B_{s}^{H}$.

(iii) The backward integral of $u(t)$ with respect to $B_{t}^{H}$ is defined as

$$
\lim _{\varepsilon \rightarrow 0} \frac{1}{\varepsilon} \int_{0}^{T} u(s)\left[\frac{B_{s-\varepsilon}^{H}-B_{s}^{H}}{\varepsilon}\right] d s,
$$

provided that the limit exists in probability, and is denoted by $\int_{0}^{T} u(s) d^{+} B_{s}^{H}$.

For the convenience of readers, some basic properties of the path-wise stochastic integrals are provided as follows:

Let $\varphi: \mathbb{R}_{+} \times \mathbb{R}_{+} \rightarrow \mathbb{R}_{+}$be defined by

$$
\varphi(t, s)=H(2 H-1)|t-s|^{2 H-2}, t, s \in \mathbb{R}_{+},
$$


where $H$ is a constant with $\frac{1}{2}<H<1$.

Let $g: \mathbb{R}_{+} \rightarrow \mathbb{R}$ be borel mesurable, define

$$
L_{\varphi}^{2}\left(\mathbb{R}_{+}\right)=\left\{g:\|g\|_{\varphi}^{2}=\int_{\mathbb{R}_{+}} \int_{\mathbb{R}_{+}} g(t) g(s) \varphi(t, s) d s d t<\infty\right\},
$$

if we equip $L_{\varphi}^{2}\left(\mathbb{R}_{+}\right)$and with the inner product

$$
<g_{1}, g_{2}>_{\varphi}=\int_{\mathbb{R}_{+}} \int_{\mathbb{R}_{+}} g_{1}(t) g_{2}(s) \varphi(t, s) d s d t, g_{1}, g_{2} \in \mathbb{R}_{+}
$$

then $L_{\varphi}^{2}\left(\mathbb{R}_{+}\right)$become respectively the separable Hilbert space.

Let $\mathcal{S}$ be the set of smooth and cyndrical random variables of the form

$$
F(\omega)=f\left(\int_{0}^{T} \psi_{1}(t) d B_{t}^{H}, \ldots, \int_{0}^{T} \psi_{n}(t) d B_{t}^{H}\right)
$$

where $n \geq 1, f \in \mathcal{C}_{b}^{\infty}\left(\mathbb{R}_{+}^{n}\right)\left(f\right.$ and all its partial derivatives are bounded) and $\psi_{i} \in \mathcal{H}$.

$\mathcal{H}$ is the completion of the mesurable functions such that $\left\|\psi_{i}\right\|^{2}<\infty$ and $\psi_{n}$, is the sequence in $\mathcal{H}$ such that $\left\langle\psi_{i}, \psi_{j}>_{\varphi}=\delta_{i j}\right.$. We denote by $\mathcal{H}$ the space of measurable functions $h$ on $[0, T]$ satisfying

$$
\|h\|_{\mathcal{H}}^{2}=\int_{0}^{T} \int_{0}^{T}|h(t) \| h(s)| \varphi(t, s) d s d t<\infty
$$

where $\mathcal{H}$ is a Banach space with the norm $\|\cdot\|_{\mathcal{H}}^{2}$.

The malliavin derivative $D_{t}^{H}$ of a smooth and cylindrical random variable $F \in \mathcal{S}$ is defined as the $\mathcal{H}$-value random variable:

$$
D_{t}^{H} F=\sum_{i=1}^{n} \frac{\partial f}{\partial x_{i}}\left(\int_{0}^{T} \psi_{1}(t) d B_{t}^{H}, \ldots, \int_{0}^{T} \psi_{n}(t) d B_{t}^{H}\right) \psi_{i}(t),
$$

then for any $p \geq 1$, the derivative operator $D_{t}^{H}$ is a closable operator from $L^{p}(\Omega)$ into $L^{p}(\Omega, \mathcal{H})$. We define the $\varphi$-derivative of $F$ :

$$
D_{t}^{\varphi} F=\int_{\mathbb{R}_{+}} \varphi(t, v) D_{v}^{H} F d v .
$$

Definition 2. The space $L_{\varphi}[0, T]$ of integrands is defined as the family of stochastic process $u(t)$ on $[0, T]$, such that $\mathbb{E} \int_{0}^{T}\|u(s)\|_{\varphi}^{2}<\infty u(t)$ is $\varphi$-differentiable, the trace of $D_{t}^{\varphi} u(t)$ exists; $0 \leq s \leq T$, $0 \leq t \leq T, \mathbb{E} \int_{0}^{T} \int_{0}^{T}\left[D_{s}^{\varphi} u(s)\right]^{2} d s d t<\infty$ and for each sequence of partition $\left(\pi_{n}, n \in \mathbb{N}\right)$ such that $\left|\pi_{n}\right| \rightarrow 0$ as $n \rightarrow 0$

$$
\sum_{i=0}^{n-1} \mathbb{E} \int_{t_{i}^{(n)}}^{t_{i+1}^{(n)}} \int_{t_{j}^{(n)}}^{t_{j+1}^{(n)}}\left|D_{s}^{\varphi} u^{\pi}\left(t_{i}^{(n)}\right) u^{\pi}\left(t_{j}^{(n)}\right)-D_{s}^{\varphi} u(t) D_{t}^{\varphi} u(s)\right| d s d t
$$

and $\mathbb{E}\left[\left\|u^{\pi}-u\right\|_{\varphi}^{2}\right]^{2}$ tend to 0 as $n \rightarrow 0$, where $\pi_{n}=t_{0}^{(n)}<t_{1}^{(n)}<\ldots<t_{n-1}^{(n)}<t_{n}^{(n)}=T$.

According to the remark 1 in [3] and Proposition 6.2.3 in [7]. Let $u(t)$ be a stochastic process in the space $\mathbb{D}^{1,2}(|\mathcal{H}|)$, and satisfies $\int_{0}^{T} \int_{0}^{T}\left|D_{s}^{H} u(s)\right|^{2 H-2} d s d t<\infty$ then we can see that symetric integral $\int_{0}^{T} u(s) d^{\circ} B_{s}^{H}$ coincides with the forward and backward integrals. 
If $u(s) \in L_{\varphi}[0, T]$, then one of the pathwise integrals exist and the following relation holds :

$$
\int_{0}^{T} u(s) d^{\circ} B_{s}^{H}=\int_{0}^{T} u(s) \diamond d B_{s}^{H}+\int_{0}^{T} D_{t}^{\varphi} u(s) d s .
$$

Lemma 1. Let $B_{t}^{H}$ be the $f B m$ with $\frac{1}{2}<H<1$ and $u(t)$ be a stochastic process in $D^{1,2}(|\mathcal{H}|) \cap$ $\left(L_{\varphi}[0, T]\right)$, then for every $T<\infty$,

$$
\mathbb{E}\left[\int_{0}^{T} u(s) d^{\circ} B_{s}^{H}\right]^{2} \leq 2 H T^{2 H-1} \mathbb{E}\left[\int_{0}^{T}|u(s)|^{2} d s\right]+4 T \mathbb{E}\left[\int_{0}^{T} D_{s}^{\varphi} u(s)\right]^{2} d s .
$$

Proof. We have:

$$
\begin{aligned}
\mathbb{E}\left[\int_{0}^{T} u(s) d^{\circ} B_{s}^{H}\right]^{2} & =\mathbb{E}\left[\int_{0}^{T} u(s) \diamond d B_{s}^{H}+\int_{0}^{T} D_{t}^{\varphi} u(s) d s\right]^{2} \\
& \leq 2 \mathbb{E}\left[\int_{0}^{T} u(s) \diamond d B_{s}^{H}\right]+2 \mathbb{E}\left[\int_{0}^{T} D_{s}^{\varphi} u(s)\right]^{2} d s \\
& \leq 2 H T^{2 H-1} \mathbb{E}\left[\int_{0}^{T}|u(s)|^{2} d s\right]+4 T \mathbb{E}\left[\int_{0}^{T} D_{s}^{\varphi} u(s)\right]^{2} d s
\end{aligned}
$$

\subsection{Hypothesis of non-Lipschitz condition}

Throughout this paper we assume that the coefficients $\mu, \sigma_{1}$ and $\sigma_{2}$, which are continuous, satisfy, for all $x, y \in \mathbb{R}^{n}$ and $t \in[0, T]$, the assumptions (A.1) and (A.2):

A.1 The functions $\mu$ and $\sigma_{1}$ have a linear growth and satisfy suitable modulus of continuity with respect to variable $x$ uniformly in $t$. Assumption A.1 means that $\mu$ and $\sigma_{1}$ satisfy:

A.1.1 $\mu(t, x) \mid \leq K(1+|x|)$

A.1.2 $|\mu(t, x)-\mu(t, y)|^{2} \leq \varrho\left(|x-y|^{2}\right)$

A.1.3 $\sigma_{1}(t, x) \mid \leq K(1+|x|)$

A.1.4 $\sigma_{1}(t, x)-\sigma_{1}(t, y) \leq \varrho\left(|x-y|^{2}\right)$,

where $\varrho$ is a concave increasing function from $\mathbb{R}_{+}$to $\mathbb{R}_{+}$such that $\varrho(0)=0$,

$\varrho(u)>0$ for $u>0$ and

$$
\int_{0^{+}} \frac{d u}{\varrho(u)}=+\infty
$$

A.2 The functions $\mu(t, 0)$ and $\sigma_{2}(t, 0)$ are locally integral with respect to $t$, and the function $\sigma_{2}$ is continuously differentiable in the first variable $t$. Assumption A.2 means that $\mu$ and $\sigma_{2}$ satisfy $\forall t \in[0, T], \mu(t,),. \sigma_{2}(t,.) \in L_{\varphi}([0, T]) \cap \mathbb{D}^{1,2}(|\mathcal{H}|)$ :

$$
\mathbb{E}|\widetilde{\mu}(t, x, y)|^{2}+\mathbb{E}\left|\widetilde{\sigma}_{2}(t, x, y)\right|^{2}+\mathbb{E}\left|D_{t}^{\varphi}\left(\widetilde{\sigma}_{2}(t, x, y)\right)\right|^{2} \leq \varrho\left(\mathbb{E}|x-y|^{2}\right),
$$

with $\varphi$ is given by $(7)$, and

$$
\left\{\begin{array}{l}
\widetilde{\sigma}_{2}(t, x, y)=\sigma_{2}(t, x)-\sigma_{2}(t, y) \\
\widetilde{\mu}(t, x, y)=\mu(t, x)-\mu(t, y)
\end{array}\right.
$$

The non-Lipschitz condition has a variety of forms [4], [27], [1] and [28]. 
Now, let define two sequences of functions $\left\{\chi_{n}(t)\right\}_{n=1,2, \ldots}$ and $\left\{\tilde{\chi}_{n, p}(t)\right\}_{n=1,2, \ldots}$ such that $\chi_{1}(t)=$ $C t, \chi_{n+1}(t)=\int_{0}^{t} \varrho_{1}\left(\chi_{n}(s)\right) d s$ and $\tilde{\chi}_{n, p}(t)=\sup _{0 \leq s \leq t} \mathbb{E}\left|X_{n+p}(s)-X_{n}(s)\right|^{2}, n=1,2, \ldots$ where $p \geq 1$ is fixed arbitrarily.

Lemma 2. (Liu, [20]) Under the non-Lipschitz condition,

$$
0 \leq \tilde{\chi}_{n, p}(t) \leq \chi_{n}(t) \leq \chi_{n-1}(t) \leq \ldots \leq \chi_{1}(t)
$$

for all positive integer $n$.

Lemma 3. There exists a positive number $G$,

$$
\begin{gathered}
\forall \mu(t, \cdot), \sigma_{2}(t, \cdot) \in L_{\varphi}([0, T]) \cap \mathbb{D}^{1,2}(|\mathcal{H}|), \\
\mathbb{E}|\mu(t, x)|^{2}+\mathbb{E}\left|\sigma_{2}(t, x)\right|^{2}+\mathbb{E}\left|D_{t}^{\varphi}\left(\sigma_{2}(t, x)\right)\right|^{2} \leq G\left(1+\mathbb{E}|x|^{2}\right) .
\end{gathered}
$$

Proof. Since $\varrho(u)$ is a concave and non-negative function, we can choose two positive constants $a>0$ and $b>0$, so that $\kappa(u) \leq a+b u$

$$
\begin{aligned}
& \mathbb{E}|\mu(t, x)|^{2}+\mathbb{E}\left|\sigma_{2}(t, x)\right|^{2}+\mathbb{E}\left|D_{t}^{\varphi}\left(\sigma_{2}(t, x)\right)\right|^{2} \\
& \leq 2 \mathbb{E}\left(|\mu(t, 0)|^{2}+\left|\sigma_{2}(t, 0)\right|^{2}+\left|D_{t}^{\varphi}\left(\sigma_{2}(t, 0)\right)\right|^{2}\right) \\
& +2 \mathbb{E}|\mu(t, x)-\mu(t, 0)|^{2}+2 \mathbb{E}\left|\sigma_{2}(t, x)-\sigma_{2}(t, 0)\right|^{2}+2 \mathbb{E}\left|D_{t}^{\varphi}\left(\sigma_{2}(t, x)\right)-\sigma_{2}(t, 0)\right|^{2} \\
& \leq 2 \sup _{0 \leq t \leq T} \mathbb{E}\left(|\mu(t, 0)|^{2}+\left|\sigma_{2}(t, 0)\right|^{2}+\left|D_{t}^{\varphi}\left(\sigma_{2}(t, 0)\right)\right|^{2}\right)+2 \varrho\left(\mathbb{E}(x)^{2}\right) \\
& \leq G\left(1+\mathbb{E}(x)^{2}\right),
\end{aligned}
$$

where

$$
G=2 \sup _{0 \leq t \leq T}\left\{\mathbb{E}\left(|\mu(t, 0)|^{2}+\left|\sigma_{2}(t, 0)\right|^{2}+\left|D_{t}^{\varphi}\left(\sigma_{2}(t, 0)\right)\right|^{2}\right)+2 a, 2 b\right\}<\infty
$$

\section{The main results}

\subsection{The MFH model framework}

Mixed Fractional Heston model is the Heston model in which the volatility Brownian and the price Brownian are replaced by the MFBM. So we first consider the Heston model which will be described in Definition 3.

Definition 3. (Heston Model, [15]) The model given by [15] as one of the most the important stochastic volatility models. In this model the volatility is a stochastic process and it is determined by the stochastic differential equation (SDE) as follows, see [21]

$$
\begin{aligned}
& d S_{t}=S_{t} \mu d t+\sqrt{V_{t}} S_{t} d B_{1, t} \\
& d V_{t}=\kappa\left(\theta-V_{t}\right) d t+\sigma \sqrt{V_{t}} d B_{2, t} \\
& d B_{1, t} \times d B_{2, t}=\rho d t
\end{aligned}
$$

where $B_{1, t}$ and $B_{2, t}$ are two Brownian motion process with correlation $\rho \in(-1,1)$ and $S$ represent the current stock price, $V$ is the volatility, $\kappa$ is the rate which $V$ reverts to $\theta, \theta$ is the long variance and $\sigma$ is the volatility of the volatility. 
Definition 4. (MFH model) Let us consider a probability space $(\Omega, F, \mathbb{P})$ on some Brownian motion $B_{i}=B_{i, t}$, fractional Brownian motion $B_{i}^{H}=B_{i, t}^{H}$, for $i=1,2$. Let $\left(F_{t}\right)_{t \geq 0}$ be a flltration generated by these three above process and $\mathbb{P}$ a risk neutral probability under the asset price process $S_{t}$ at time $t \geq 0$. Let $V_{t}$ be stochastic volatility process at time $t \geq 0$. In the Heston model, if we substitute $B_{i, t}$ by $M_{i}^{H}=M_{i, t}^{H}$ then, we obtain a Mixed Fractional Heston model and its SDE's is given by

$$
\left\{\begin{array}{l}
d S_{t}=S_{t} \mu d t+\sqrt{V_{t}} S_{t} d M_{1, t}^{H} \\
d V_{t}=\kappa\left(\theta-V_{t}\right) d t+\sigma \sqrt{V_{t}} d M_{2, t}^{H}
\end{array}\right.
$$

with $d M_{1, t}^{H} \times d M_{2, t}^{H}=\rho\left(a^{2} d t+b^{2} d t^{2 H}\right), \rho \in(0,1)$. i.e.

$$
d S_{t}=S_{t} \mu d t+a \sqrt{V_{t}} S_{t} d B_{t, 1}+b \sqrt{V_{t}} S_{t} d B_{1, t}^{H}
$$

and

$$
d V_{t}=\kappa\left(\theta-V_{t}\right) d t+a \sigma \sqrt{V_{t}} S_{t} d B_{t, 2}+b \sigma \sqrt{V_{t}} S_{t} d B_{2, t}^{H}
$$

where $\kappa$ control the speed of mean reversion of the volatility and $\theta$ is the long-run mean of the volatility, $\sigma$ is the volatility of $V_{t}$ process. $S_{0}$ and $V_{0}$ are spot asset price and spot variance respectively.

$V_{t}$ is strictly positive when $2 \kappa \theta \geq \sigma^{2}$ and non-negative when $0 \leq 2 \kappa \theta<\sigma^{2}$ (Feller condition). $\rho$ is the coefficient of correlation between $B_{i}$, fractional Brownian motion $B_{i}^{H}$.

\subsection{Simulation of MFH model}

Euler's scheme is the simplest way to discritize the stochastic differential equations [16]. We perform Euler discretisation on the MFH model. The Euler discretization can be used to approximate the asset path of the stock price on a discrete time grid [16]. Let $S_{t}$ be an asset price which implies in (25) and $V_{t}$ satisfying $(26)$. Let $\Lambda=\left\{t_{0}, t_{1}, \ldots, t_{N}\right\}$ be a partition of the interval $[0, T]$. i.e $0<t_{0}<t_{1}<\ldots<t_{N}=T$ then, we have for all $0 \leq j \leq N-1$ and $i=1,2$,

$$
\begin{aligned}
& S_{j+1}=S_{j}+\mu S_{j} \Delta t+\sqrt{V_{j}} S_{j} \Delta M_{i j}^{H} \\
& V_{j+1}=V_{j}+\kappa\left(\theta-V_{j}\right) \Delta t+\sigma \sqrt{V_{j}} \Delta M_{i j}^{H} .
\end{aligned}
$$

We have the following formula, for all $0 \leq j \leq N-1, t_{j}=j \Delta t$ and $i=1,2,3$.

$$
\Delta M_{i j}^{H}=M_{i}^{H}\left(t_{j+1}\right)-M_{i}^{H}\left(t_{j}\right)
$$

and

$$
\Delta M_{i j}^{H} \sim \mathcal{N}\left(0, a^{2} \Delta t+b^{2} \Delta t^{2 H}\right) .
$$

According to the central limit theorem, we have

$$
\Delta M_{i j}^{H}=\mathcal{N}\left(0, a \mathcal{Z}_{i} \sqrt{\Delta t}+b \mathcal{Z}_{i} \sqrt{\Delta t^{2 H}}\right)
$$

and

$$
\mathcal{Z}_{i} \sim \mathcal{N}(0,1)
$$

Therefore, we have

$$
S_{j+1}=S_{j}+\mu S_{j} \Delta t+a \sqrt{V_{j} \Delta t} S_{j} Z_{1}+b S_{j} Z_{1} \sqrt{V_{j} \Delta t^{2 H}},
$$


and

$$
V_{j+1}=V_{j}+\kappa\left(\theta-V_{j}\right) \Delta t+a \sigma \sqrt{V_{j} \Delta t} \phi_{1}+b \sigma \phi_{1} \sqrt{V_{j} \Delta t^{2 H}}
$$

where the correlated normal variables, $\phi_{1}=\rho \mathcal{Z}_{1}+\sqrt{1-\rho^{2}} \mathcal{Z}_{2}$ generated by the Cholesky's method.

The parameters of the MFH model are taken from [24] and are presented in the following table 1. The asset price has been estimated under the MFH model, where the parameter of the option model is given by the table 1 defined by:

\begin{tabular}{cccccccccc}
\hline $\mathrm{T}$ & $\rho$ & $S_{0}$ & $V_{0}$ & $\mu$ & $\sigma$ & $\kappa$ & $\theta$ & $\Delta t$ & $\mathrm{E}$ \\
\hline 2 & 0.26 & 0.04 & 0.04 & 0.07 & 0.04 & 2 & 3 & 0.001 & 100 \\
\hline
\end{tabular}

Table 1: Parameter of MFH model

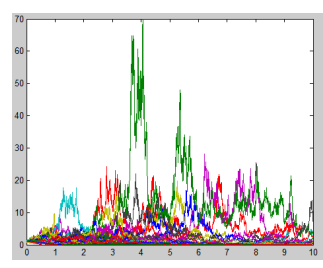

(a) $[\mathrm{H}=0.76]$

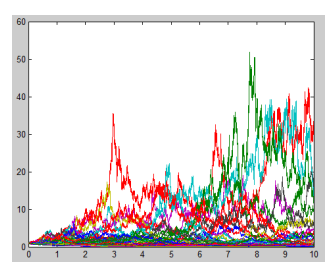

(b) $[\mathrm{H}=0.77]$

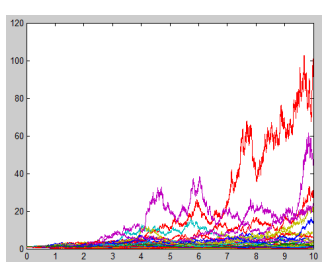

(c) $[\mathrm{H}=0.78]$

Figure 1: Simulated asset paths of MFH model.

In Figure 1 above, we see 80 simulated paths for the asset price with different Hurst parameter $H$ such that $H>\frac{3}{4}$. Figure 1 below shows that increasing or decreasing the hurst parameters affects the future price of the asset so that, by increasing the Hurst parameter, the difference between expected lowest price and the highest price will be increased.

The simulation of the MFH model is given by the following algorithm

\section{Algorithm 1. MFH model simulation process.}

(i) Set $\Delta=\frac{t}{N}$.

(ii) For $i=1$ to number of simulation.

(iii) Generate independent standard normal variables, $Z_{j} \sim \mathcal{N}(0,1), j=1, \ldots, N$.

(iv) $\operatorname{Set} S_{j+1} \leftarrow S_{j}+\mu S_{j} \Delta t+a\left(V_{j} \Delta t\right)^{\frac{1}{2}} S_{j} Z_{1}+b S_{j} Z_{1}\left(V_{j} \Delta t^{2 H}\right)^{\frac{1}{2}}$.

(v) For $V_{j+1} \leftarrow V_{j}+\kappa\left(\theta-V_{j}\right) \Delta t+a \sigma\left(V_{j} \Delta t\right)^{\frac{1}{2}} \phi_{1}+b \sigma \phi_{1}\left(V_{j} \Delta t^{2 H}\right)^{\frac{1}{2}}$.

(vi) End For. 


\subsection{Existence and Uniqueness}

Now we will discuss the solutions for non-Lipschitz SDE's with Brownian motion, fBm for each equation defined in (58) by using an iteration of Picard [12]. Let $X_{0}(t)=\zeta$ be a random variable with $\mathbb{E}|\zeta|^{2}<+\infty$. In the general case, we construct an approximative sequence of stochastic process $\left\{X_{p}(t)\right\}_{p \geq 1}$ as follows

$$
\begin{aligned}
X_{p}(t) & =\zeta+\int_{0}^{t} \mu\left(s, X_{p-1}(s)\right) d s+\int_{0}^{t} \sigma_{1}\left(s, X_{p-1}(s)\right) d B_{s} \\
& +\int_{0}^{t} \sigma_{2}\left(s, X_{p-1}(s)\right) d B_{s}^{H} .
\end{aligned}
$$

Theorem 1. Under the assumptions $\boldsymbol{A . 1}$ and $\boldsymbol{A . 2}$, the path-wise uniqueness holds for $(3), t \in$ $[0, T]$.

Proof. Let $Y(t)$ and $Z(t)$ be two solutions of (34) and $Y(0)=Z(0)$, we have

$$
\begin{aligned}
Y(t)-Z(t) & =\int_{0}^{t} \widetilde{\mu}(s) d s+\int_{0}^{t} \tilde{\sigma}_{1}(s) d B_{s}+\int_{0}^{t} \widetilde{\sigma}_{2}(s) d^{\circ} B_{s}^{H} \\
& =I_{\mu}(t)+I_{\sigma_{1}}(t)+I_{\sigma_{2}}(t)
\end{aligned}
$$

with

$$
\left\{\begin{array}{l}
I_{\mu}(t)=\int_{0}^{t} \widetilde{\mu}(s) d s \\
I_{\sigma_{1}}(t)=\int_{0}^{t} \widetilde{\sigma}_{1}(s) d B_{s} \\
I_{\sigma_{2}}(t)=\int_{0}^{t} \widetilde{\sigma}_{2}(s) d^{\circ} B_{s}^{H}
\end{array}\right.
$$

where

$$
\left\{\begin{array}{l}
\widetilde{\mu}(s)=\mu(s, Y(s))-\mu(s, Z(s)) \\
\widetilde{\sigma}_{1}(s)=\sigma_{1}(s, Y(s))-\sigma_{1}(s, Z(s)) \\
\widetilde{\sigma}_{2}(s)=\sigma_{2}(s, Y(s))-\sigma_{2}(s, Z(s))
\end{array}\right.
$$

By employing the following inequality

$$
\forall a_{1}, a_{2}, a_{3} \in \mathbb{R}, \quad\left|a_{1}+a_{2}+a_{3}\right|^{2} \leq 3\left|a_{1}\right|^{2}+3\left|a_{2}\right|^{2}+3\left|a_{3}\right|^{2} .
$$

It follows that

$$
|Y(t)-Z(t)|^{2} \leq 3\left|\int_{0}^{t} \widetilde{\mu}(s) d s\right|^{2}+3\left|\int_{0}^{t} \widetilde{\sigma}_{1}(s) d B_{s}\right|^{2}+3\left|\int_{0}^{t} \widetilde{\sigma}_{2}(s) d^{\circ} B_{s}^{H}\right|^{2} .
$$

We observe

$$
\mathbb{E}|Y(t)-Z(t)|^{2} \leq 3 \mathbb{E}\left|I_{\mu}(t)\right|^{2}+3 \mathbb{E}\left|I_{\sigma_{1}}(t)\right|^{2}+3 \mathbb{E}\left|I_{\sigma_{2}}(t)\right|^{2} .
$$

We have to estimate $\mathbb{E}\left|I_{\mu}(t)\right|^{2}, \mathbb{E}\left|I_{\sigma_{1}}(t)\right|^{2}$ and $\mathbb{E}\left|I_{\sigma_{2}}(t)\right|^{2}$.

According to the Ito's Isometry we have

$$
\mathbb{E}\left|\int_{0}^{t} \widetilde{\sigma}_{1}(s) d B_{s}\right|^{2}=\mathbb{E} \int_{0}^{t}\left|\widetilde{\sigma}_{1}(s)\right|^{2} d s .
$$

By using Fubini's Theorem, we have

$$
\mathbb{E} \int_{0}^{t}\left|\widetilde{\sigma}_{1}(s)\right|^{2} d s=\int_{0}^{t} \mathbb{E}\left|\widetilde{\sigma}_{1}(s)\right|^{2} d s
$$


Using the linear growth assumption in (A.1.4), it is easy to see that

$$
\left|\widetilde{\sigma}_{1}(s)\right|^{2} \leq \varrho\left(|Y(s)-Z(s)|^{2}\right)
$$

and then

$$
\mathbb{E}\left|\widetilde{\sigma}_{1}(s)\right|^{2} \leq \mathbb{E}\left[\varrho\left(|Y(s)-Z(s)|^{2}\right)\right] .
$$

According to the Jensen's Inequality, we have

$$
\mathbb{E}\left[\varrho\left(|Y(s)-Z(s)|^{2}\right)\right] \leq \varrho\left(\mathbb{E}|Y(s)-Z(s)|^{2}\right)
$$

it follows that

$$
\int_{0}^{t} \mathbb{E}\left|\widetilde{\sigma}_{1}(s)\right|^{2} d s \leq \int_{0}^{t} \varrho\left(\mathbb{E}|Y(s)-Z(s)|^{2}\right) d s
$$

Therefore,

$$
\mathbb{E}\left|I_{\sigma_{1}}(t)\right|^{2} \leq \int_{0}^{t} \varrho\left(\mathbb{E}|Y(s)-Z(s)|^{2}\right) d s .
$$

Using the simple estimation, we have

$$
\mathbb{E}\left|I_{\mu}(t)\right|^{2}=\mathbb{E}\left|\int_{0}^{t} \widetilde{\mu}(s) d s\right|^{2} \leq T \mathbb{E} \int_{0}^{t}|\widetilde{\mu}(s)|^{2} d s,
$$

and by Fubini's Theorem, we have

$$
\mathbb{E}\left|I_{\mu}(t)\right|^{2} \leq T \int_{0}^{t} \mathbb{E}|\widetilde{\mu}(s)|^{2} d s .
$$

Consequently

$$
\mathbb{E}\left|I_{\mu}(t)\right|^{2} \leq 8 T \int_{0}^{t} \mathbb{E}|\widetilde{\mu}(s)|^{2} d s .
$$

We know that $B_{t}^{H}$ is the fBm with $\frac{1}{2}<H<1$ and $\widetilde{\sigma}_{2}(t)$ is a stochastic process in $D^{1,2}(|\mathcal{H}|) \cap$ $\left(L_{\varphi}[0, T]\right)$, for every $t \in[0, T]$, we have from lemma 1 that

$$
\mathbb{E}\left[\int_{0}^{t} \widetilde{\sigma}_{2}(s) d^{\circ} B_{s}^{H}\right]^{2} \leq 2 H t^{2 H-1} \mathbb{E}\left[\int_{0}^{t}\left|\widetilde{\sigma}_{2}(s)\right|^{2} d s\right]+4 t \mathbb{E}\left[\int_{0}^{t} D_{s}^{\varphi} \widetilde{\sigma}_{2}(s)\right]^{2} d s .
$$

The inequality (50) implies that

$$
\mathbb{E}\left|I_{\sigma_{2}}(t)\right|^{2} \leq 8 T \int_{0}^{t}\left[\mathbb{E}\left|\widetilde{\sigma}_{2}(s)\right|^{2}+\mathbb{E}\left|D_{s}^{\varphi} \widetilde{\sigma}_{2}(s)\right|^{2}\right] d s .
$$

By combining (51) and (49), we obtain

$$
\mathbb{E}\left|I_{\mu}(t)\right|^{2}+\mathbb{E}\left|I_{\sigma_{2}}(t)\right|^{2} \leq 8 T \int_{0}^{t}\left[\mathbb{E}|\widetilde{\mu}(s)|^{2} d s+\mathbb{E}\left|\widetilde{\sigma}_{2}(s)\right|^{2}+\mathbb{E}\left|D_{s}^{\varphi} \widetilde{\sigma}_{2}(s)\right|^{2}\right] d s .
$$

Using the inequality (17) of the assumption (A.2), we obtain

$$
\mathbb{E}\left|I_{\mu}(t)\right|^{2}+\mathbb{E}\left|I_{\sigma_{2}}(t)\right|^{2} \leq 8 T \int_{0}^{t} \varrho\left(\mathbb{E}|Y(t)-Z(t)|^{2}\right) d s .
$$


The inequality (46) and (53) give

$$
\mathbb{E}\left|I_{\sigma_{1}}(t)\right|^{2}+\mathbb{E}\left|I_{\mu}(t)\right|^{2}+\mathbb{E}\left|I_{\sigma_{2}}(t)\right|^{2} \leq(1+8 T) \int_{0}^{t} \varrho\left(\mathbb{E}|Y(s)-Z(s)|^{2}\right) d s .
$$

The inequality (39) give

$$
\mathbb{E}|Y(t)-Z(t)|^{2} \leq(3+24 T) \int_{0}^{t} \varrho\left(\mathbb{E}|Y(s)-Z(s)|^{2}\right) d s .
$$

Noticing that from (16), the inequality (55) implies that $\mathbb{E}|Y(t)-Z(t)|^{2}=0, \forall t \in[0, T]$. Since $T>0$ is an arbitrary, $Y(t) \equiv Z(t), \forall t \in[0, T]$. Thus the path-wise uniqueness holds for (3).

To prove the existence of Theorem 1, we show that under the non-Lipschitz condition,

$$
\lim _{n, i \rightarrow \infty} \sup _{0 \leq t \leq T} \mathbb{E}\left|X_{p}(t)-X_{i}(t)\right|^{2}=0 .
$$

We call $\left\{X_{p}(\cdot)\right\}_{p \geq 1}$ a Cauchy sequence which $X(\cdot)$ is its limit. By letting $p \rightarrow \infty$ in (34), we deduce that the solution to (1) exist. We fix $p \geq 1$ arbitrary and define two sequences of functions $\left\{\chi_{n}(t)\right\}_{n \geq 1}$ and $\left\{\widetilde{\chi}_{n, p}(t)\right\}_{n \geq 1}$ where

$$
\left\{\begin{array}{l}
\chi_{1}(t)=C t \\
\chi_{n+1}(t)=\int_{0}^{t} \varrho_{1}\left(\chi_{n}(s)\right) d s \\
\widetilde{\chi}_{n, p}(t)=\sup _{0 \leq t \leq T} \mathbb{E}\left|X_{p}(t)-X_{i}(t)\right|^{2} .
\end{array}\right.
$$

By Lemma 2, we observe that $\left(\chi_{n}(t)\right)$ decreases when $n \rightarrow \infty$ and is nonnegative function on $t \in[0, T]$, therefore, we define $\chi(t)$ as limit of $\left(\chi_{n}(t)\right)$, we have $\chi(0)=0$ and $\chi(t)$ is a continuous function on $t \in[0, T]$. Or $\chi(t)=\lim _{n \rightarrow \infty} \chi_{n}(t)$, we have

$$
\lim _{n \rightarrow \infty} \chi_{n+1}(t)=\lim _{n \rightarrow \infty} \int_{0}^{t} \varrho_{1}\left(\chi_{n}(s)\right) d s=\int_{0}^{t} \varrho_{1}(\chi(s)) d s .
$$

Since $\chi(0)=0$ and $\int_{0^{+}} \frac{d u}{\varrho(u)}=+\infty$, we say that (58) implies $\chi(t)=0$, therefore we get from the inequality (19)

$$
\begin{aligned}
0 \leq \lim _{n, p \rightarrow \infty} \chi_{n+1}(t) & =\lim _{n \rightarrow \infty} \sup _{0 \leq t \leq T} \mathbb{E}\left|X_{p}(t)-X_{i}(t)\right|^{2} \\
& =\lim _{n, p \rightarrow \infty} \widetilde{\chi}_{n, p}(T) \\
& \leq \lim _{n \rightarrow \infty} \widetilde{\chi}_{n}(T)=0
\end{aligned}
$$

namely, $\lim _{n, i \rightarrow \infty} \sup _{0 \leq t \leq T} \mathbb{E}\left|X_{n}(t)-X_{i}(t)\right|^{2}=0$.

Remark 1. The asset price $S_{t}$ satisfy

$$
d S_{t}=S_{t} \mu d t+a \sqrt{V_{t}} S_{t} d B_{t, 1}+b \sqrt{V_{t}} S_{t} d B_{1, t}^{H} .
$$

With respect to the above equation defined by (60), we can say that the stock price equation of the MFH model has a unique solution. 
Let $V_{0}(t)=\zeta$ be a random variable with $\mathbb{E}|\zeta|^{2}<+\infty$.

We also construct an approximative sequence of stochastic process $\left\{V_{t}^{n}\right\}_{n \geq 1}$ as follows

$$
\begin{aligned}
V_{t}^{n} & =\zeta+\int_{0}^{t} \kappa\left(\theta-V_{s}^{n-1}\right) d s+\int_{0}^{t} \sigma a \sqrt{V_{s}^{n-1}} d B_{s} \\
& +\int_{0}^{t} \sigma b \sqrt{V_{s}^{n-1}} d B_{s}^{H} .
\end{aligned}
$$

Theorem 2. Under the assumptions $\boldsymbol{A . 1}$ and A.2, the volatility of the mixed Heston model has a unique positive solution $V_{t}$, where $t \in[0, T]$ and $T=\inf \left\{t>0 \mid X_{t}=0\right\}$.

Proof. We have

$$
V_{t}=\zeta+\int_{0}^{t} \kappa\left(\theta-V_{s}\right) d s+\int_{0}^{t} \sigma \sqrt{V_{s}} d M_{s}^{H} .
$$

Suppose that for some initial value $\zeta$ there are two continous solutions $V_{t}$ and $\widetilde{V}_{t}$ satisfy $(62)$, then the difference satisfies

$$
\begin{aligned}
V_{t}-\widetilde{V}_{t} & =\int_{0}^{t} \psi_{1}(s) d s+\sigma a \int_{0}^{t} \psi_{2}(s) d B_{s}+\sigma b \int_{0}^{t} \psi_{3}(s) d B_{s}^{H} \\
& =J_{1}(t)+J_{2}(t)+J_{3}(t)
\end{aligned}
$$

where

$$
\left\{\begin{array}{l}
J_{1}(t)=\int_{0}^{t} \psi_{1}(s) d s \\
J_{2}(t)=\sigma a \int_{0}^{t} \psi_{2}(s) d B_{s} \\
J_{3}(t)=\sigma b \int_{0}^{t} \psi_{3}(s) d B_{s}^{H}
\end{array}\right.
$$

with

We observe that

$$
\left\{\begin{array}{l}
\psi_{1}(t)=-\kappa\left(V_{s}-\widetilde{V}_{s}\right) d s \\
\psi_{2}(t)=\sigma a\left(\sqrt{V_{s}}-\sqrt{\widetilde{V}_{s}}\right) \\
\psi_{3}(t)=\sigma b\left(\sqrt{V_{s}}-\sqrt{\widetilde{V}_{s}}\right)
\end{array}\right.
$$

$$
\mathbb{E}\left|V_{t}-\widetilde{V}_{t}\right|^{2} \leq 3 \mathbb{E}\left|J_{1}(t)\right|^{2}+3 \mathbb{E}\left|J_{2}(t)\right|^{2}+3 \mathbb{E}\left|J_{3}(t)\right|^{2}
$$

Now we have to estimated $\mathbb{E}\left|J_{i}(t)\right|^{2}, i=1,2,3$.

By letting

$$
\left.\epsilon=\min \left\{\sqrt{V_{t}}\right)+\sqrt{\widetilde{V}_{t}}>0 \mid t \in[0, T]\right\},
$$

according to the Ito isometry, we get

$$
\mathbb{E}\left|J_{2}(t)\right|^{2}=\mathbb{E} \int_{0}^{t}\left|\psi_{2}(s)\right|^{2} d s .
$$

By Fubini's Theorem

$$
\mathbb{E}\left|J_{2}(t)\right|^{2}=\int_{0}^{t} \mathbb{E}\left|\psi_{2}(s)\right|^{2} d s .
$$

Using the assumption in (A.1.2), we have

$$
\mathbb{E}\left|\psi_{2}(s)\right|^{2} \leq \frac{\sigma^{2} a^{2}}{\epsilon^{2}} \mathbb{E}\left(\varrho\left|\left(V_{s}-\widetilde{V}_{s}\right)\right|^{2}\right),
$$


and the Jensen's inequality give

$$
\mathbb{E}\left|\psi_{2}(s)\right|^{2} \leq \frac{\sigma^{2} a^{2}}{\epsilon^{2}} \varrho\left(\mathbb{E}\left|\left(V_{s}-\widetilde{V}_{s}\right)\right|^{2}\right) .
$$

Therefore

$$
\mathbb{E}\left|J_{2}(t)\right|^{2} \leq \frac{\sigma^{2} a^{2}}{\epsilon^{2}} \int_{0}^{t} \varrho\left(\mathbb{E}\left|\left(V_{s}-\widetilde{V}_{s}\right)\right|^{2}\right) d s,
$$

using the simple estimation and Fubini's Theorem, we have

$$
\mathbb{E}\left|J_{1}(t)\right|^{2} \leq T \int_{0}^{t} \mathbb{E}\left|\psi_{1}(s)\right|^{2} d s .
$$

Let $M=\min \left\{\frac{1}{\epsilon^{2}}, 1\right\}$, we have

$$
\mathbb{E}\left|J_{1}(t)\right|^{2} \leq 8 T M \int_{0}^{t} \mathbb{E}\left|\psi_{1}(s)\right|^{2} d s
$$

We know that $B_{t}^{H}$ is the fBm with $\frac{1}{2}<H<1$ and $\psi_{3}(t)$ is a stochastic process in $D^{1,2}(|\mathcal{H}|) \cap$ $\left(L_{\varphi}[0, T]\right)$, for every $t \in[0, T]$, we have from lemma 1 that

$$
\mathbb{E}\left[\int_{0}^{t} \psi_{3}(s) d^{\circ} B_{s}^{H}\right]^{2} \leq \frac{1}{\epsilon^{2}}\left[2 H t^{2 H-1} \mathbb{E}\left[\int_{0}^{t}\left|\widetilde{\psi}_{3}(s)\right|^{2} d s\right]+4 t \mathbb{E}\left[\int_{0}^{t} D_{s}^{\varphi} \widetilde{\psi}_{3}(s)\right]^{2} d s\right]
$$

with $\widetilde{\psi}_{3}(s)=\sigma c\left(V_{s}-\widetilde{V}_{s}\right)$.

The inequality (75) implies that

$$
\mathbb{E}\left|J_{3}(t)\right|^{2} \leq 8 T M \int_{0}^{t}\left[\mathbb{E}\left|\widetilde{\psi}_{3}(s)\right|^{2}+\mathbb{E}\left|D_{s}^{\varphi} \widetilde{\psi}_{3}(s)\right|^{2}\right] d s .
$$

By combining (76) and (74), we obtain

$$
\mathbb{E}\left|J_{1}(t)\right|^{2}+\mathbb{E}\left|J_{3}(t)\right|^{2} \leq 8 T M \int_{0}^{t}\left[\mathbb{E}\left|\psi_{1}(s)\right|^{2} d s+\mathbb{E}\left|\psi_{3}(s)\right|^{2}+\mathbb{E}\left|D_{s}^{\varphi} \psi_{3}(s)\right|^{2}\right] d s .
$$

Using the inequality (17) of the assumption (A.2), we obtain

$$
\mathbb{E}\left|J_{1}(t)\right|^{2}+\mathbb{E}\left|J_{2}(t)\right|^{2} \leq 8 T M \int_{0}^{t} \varrho\left(\mathbb{E}\left|V_{s}-\widetilde{V}_{s}\right|^{2}\right) d s .
$$

The inequality (72) and (78) give

$$
\mathbb{E}\left|J_{1}(t)\right|^{2}+\mathbb{E}\left|J_{2}(t)\right|^{2}+\mathbb{E}\left|J_{3}(t)\right|^{2} \leq M\left(\sigma^{2} a^{2}+8 T\right) \int_{0}^{t} \varrho\left(\mathbb{E}\left|V_{s}-\widetilde{V}_{s}\right|^{2}\right) d s .
$$

The inequality (66) give

$$
\mathbb{E}\left|V_{t}-\widetilde{V}_{t}\right|^{2} \leq 3 M\left(\sigma^{2} a^{2}+8 T\right) \int_{0}^{t} \varrho\left(\mathbb{E}\left|V_{s}-\widetilde{V}_{s}\right|^{2}\right) d s .
$$

Noticing that from (16), the inequality (80) implies that $\mathbb{E}\left|V_{t}-\widetilde{V}_{t}\right|^{2}=0, \forall t \in[0, T]$. Since $T>0$ is an arbitrary, $V_{t} \equiv \widetilde{V}_{t}, \forall t \in[0, T]$. Thus the path-wise uniqueness holds for (3). To show the existence, we proceed in the same way as Theorem 1. 
Theorem 3. If $V_{t}$ is the solution of (62), then $\mathbb{E}\left(\left|V_{t}\right|^{2}\right)<\infty$.

A.2,

Proof. To prove the existence of Theorem 3, we show that under the assumptions A.1 and

$$
\mathbb{E}\left|V_{t}^{n}\right|^{2}<\infty
$$

where for all $n \geq 1, V_{t}^{n}$ verify (61). By induction, we have for $n=1$, from Lemma 1 , and Lemma 15 ,

$$
\begin{aligned}
\mathbb{E}\left|V_{t}^{1}\right|^{2} & \leq 3 \mathbb{E}|\zeta|^{2}+3 \mathbb{E}\left|\int_{0}^{t} \kappa\left(\theta-V_{s}^{0}\right) d s\right|^{2} \\
& +3 \mathbb{E}\left|\int_{0}^{t} \bar{\sigma}_{1}\left(s, \sqrt{V_{s}^{0}}\right) d B_{s}\right|^{2}+3 \mathbb{E}\left|\int_{0}^{t} \bar{\sigma}_{2}\left(s, \sqrt{V_{s}^{0}}\right) d B_{s}^{H}\right|^{2} \\
& \leq 3 \mathbb{E}|\zeta|^{2}+3 \kappa^{2} \theta^{2} t^{2}+12 T \int_{0}^{t}\left[\mathbb{E}\left|\kappa\left(s, V_{s}^{0}\right)\right|^{2}+\xi_{1}\right] d s
\end{aligned}
$$

with

$$
\xi_{1}=\mathbb{E}\left|\bar{\sigma}_{2}\left(s, V_{s}^{0}\right)\right|^{2}+\mathbb{E}\left|D_{s}^{\varphi} \bar{\sigma}_{2}\left(s, V_{s}^{0}\right)\right|^{2} .
$$

Let $\bar{\epsilon}=\min _{0 \leq t \leq T}\left\{1 ; \frac{1}{\sqrt{V_{t}^{0}}}\right\}$, we have

$$
\mathbb{E}\left|V_{t}^{1}\right|^{2} \leq 3 \mathbb{E}|\zeta|^{2}+3 \kappa^{2} \theta^{2} t^{2}+12 T \bar{\epsilon}^{2} \int_{0}^{t}\left[\mathbb{E}\left|\kappa\left(s, V_{s}^{0}\right)\right|^{2}+\xi_{1}\right] d s .
$$

Moreover

$$
\mathbb{E}\left|\kappa\left(s, V_{s}^{0}\right)\right|^{2}+\xi_{1} \leq G\left(1+\mathbb{E}\left|V_{s}^{0}\right|^{2}\right),
$$

with $G=\max \left[2 \sup _{0 \leq t \leq T} \mathbb{E}\left(\left|\kappa V_{s}^{0}\right|^{2}+\bar{\xi}_{1}\right)+2 a_{1}, 2 b_{1}\right]$, where

$$
\bar{\xi}_{1}=\left|\bar{\sigma}_{2}(s, 0)\right|^{2}+\left|D_{s}^{\varphi} \bar{\sigma}_{2}(s, 0)\right|^{2} .
$$

Therefore, we get

$$
\mathbb{E}\left|V_{t}^{0}\right|^{2} \leq 3 \mathbb{E}|\zeta|^{2}+3 \kappa^{2} \theta^{2} t^{2}+12 T G \bar{\epsilon}^{2} \int_{0}^{t}\left(1+\mathbb{E}\left|V_{s}^{0}\right|^{2}\right) d s .
$$

As we know that $V_{s}^{0}=\zeta$, then we have

$$
\mathbb{E}\left|V_{t}^{1}\right|^{2} \leq 3 \mathbb{E}|\zeta|^{2}+3 \kappa^{2} \theta^{2} t^{2}+12 T G \bar{\epsilon}^{2} t\left(1+\mathbb{E}|\zeta|^{2}\right)
$$

i.e

$$
\mathbb{E}\left|V_{t}^{1}\right|^{2} \leq 3 \mathbb{E}|\zeta|^{2}+3 \kappa^{2} \theta^{2} T^{2}+12 G T^{2} \bar{\epsilon}^{2}\left(1+\mathbb{E}|\zeta|^{2}\right)<\infty .
$$

The relation (87) is true. Now assume that for all $n$,

$$
\mathbb{E}\left|V_{t}^{n}\right|^{2} \leq \bar{A}+3 \mathbb{E}|\zeta|^{2}\left[\sum_{i=0}^{n} \frac{(12 G T)^{i}}{i !} t^{i}+\sum_{i=1}^{p} \frac{(12 G T)^{i}}{i !} t^{i}\right],
$$


with $\bar{A}=3 \mathbb{E}|\zeta|^{2}+3 \kappa^{2} \theta^{2} T^{2}+12 G T^{2}$. Then we have, for $n+1$

$$
\begin{aligned}
\mathbb{E}\left|V_{t}^{n+1}\right|^{2} & \leq 3 \mathbb{E}|\zeta|^{2}+3 \kappa^{2} \theta^{2} t^{2}+12 G T \int_{0}^{t}\left(1+\mathbb{E}\left|V_{t}^{n}\right|^{2}\right) d s \\
& +12 G T \int_{0}^{t}\left(1+\mathbb{E}\left|V_{t}^{n}\right|^{2}\right) d s \\
& \leq 3 \mathbb{E}|\zeta|^{2}+3 \kappa^{2} \theta^{2} t^{2}+12 G T \int_{0}^{t}\left(1+\mathbb{E}\left|V_{t}^{n}\right|^{2}\right) d s \\
& \leq \bar{A}+12 G T \int_{0}^{t}\left(\mathbb{E}\left|V_{s}^{n}\right|^{2}\right) d s \\
& \leq \overline{\bar{A}}+3 \mathbb{E}|\zeta|^{2} \int_{0}^{t}\left[\sum_{i=0}^{n} \frac{(12 G T)^{i+1}}{i !} s^{i}+\sum_{i=1}^{p} \frac{(12 G T)^{i+1}}{i !} s^{i}\right] d s \\
& \leq \overline{\bar{A}}+3 \mathbb{E}|\zeta|^{2} \int_{0}^{t}\left[\sum_{i=1}^{n+1} \frac{(12 G T)^{i}}{i !} s^{i}+\sum_{i=2}^{p+1} \frac{(12 G T)^{i}}{i !} s^{i}\right] d s \\
& \leq \overline{\bar{A}}+3 \mathbb{E}|\zeta|^{2} \int_{0}^{t}\left[\sum_{i=1}^{n+1} \frac{(12 G T)^{i}}{i !} s^{i}+\sum_{i=2}^{p+1} \frac{(12 G T)^{i}}{i !} s^{i}\right] d s \\
& \leq \overline{\bar{A}}+3 \mathbb{E}|\zeta|^{2}\left[\sum_{i=1}^{n+1} \frac{(12 G T)^{i}}{i !} t^{i}+\sum_{i=2}^{p+1} \frac{(12 G T)^{i}}{i !} t^{i}\right] \\
, &
\end{aligned}
$$

with $\overline{\bar{A}}=\bar{A}\left(1+12 G T^{2}\right)$.

Hence

$$
\mathbb{E}\left|V_{t}^{n+1}\right|^{2} \leq \bar{A}\left(1+12 G T^{2}\right)+3 \mathbb{E}|\zeta|^{2}\left[\sum_{i=1}^{n+1} \frac{(12 G T)^{i}}{i !} t^{i}+\sum_{i=2}^{p+1} \frac{(12 G T)^{i}}{i !} t^{i}\right] .
$$

Or $e^{x} \simeq \sum_{i=1}^{n} \exp (x)$, we have

$$
\begin{aligned}
\mathbb{E}\left|V_{t}^{n+1}\right|^{2} & \leq 3 \mathbb{E}|\zeta|^{2}\left(1+12 G T^{2}\right)+3 \kappa^{2} \theta^{2} T^{2}\left(1+12 G T^{2}\right) \\
& +12 G T^{2}\left(1+12 G T^{2}\right)+3 \mathbb{E}|\zeta|^{2} \exp \left(12 G T^{2}\right)<\infty
\end{aligned}
$$

We can conclude that $\mathbb{E}\left|V_{t}^{n}\right|^{2}<\infty$ and therefore $\mathbb{E}\left(\left|V_{t}\right|^{2}\right)<\infty$.

Remark 2. If $S_{t}$ satisfy the stock price of the $M F H$ model, then $\mathbb{E}\left|S_{t}\right|^{2}<\infty$.

\section{American put option under MFH model}

In mathematical finance, Monte Carlo simulation methods are best ways for pricing the American option. The benefit of the Monte Carlo simulation method is to trade with dependent options. This method can simulate the underlying asset price path by path, then obtain the payoff associated with the data for each simulated path and using the average discounted payoff to approach the expected discounted payoff which is the value of path dependent option. Least Squares Monte Carlos method (in short LSM) is more suitable for problems in higher dimensions than oder comparable Monte Carlos Method [6], [8]. 


\subsection{LSM Algorithm}

In the LSM approach, in order to have a better price, we only recognize the in-the-money paths. The LSM algorithm is given as follows,

Algoritheorem 2. LSM algorithm

(i)

(ii)

(xvi)
Set $S_{j}$ MFH model Asset Path.

If $S_{j}<E$.

Set $\operatorname{cashflow}(j)=\left(E-S_{j}\right) e^{-r \delta t}, \mathrm{j}=1, \ldots$, number of paths.

Else $\operatorname{cashflow}(\mathrm{j})=0$.

End if.

For $\mathrm{j}=\mathrm{N}-1:-1: 1$.

Set index $=f i n d\left(E-S_{j}>0\right)$.

Set $X=\left[\right.$ ons $($ size $($ index $)) S($ index $\left.)(S(\text { index }))^{2}\right]$.

Set $B=\left(X^{T} X\right)^{\frac{1}{2}} X$ cashflow(index $)$.

Set conditional $\exp =X B$.

If conditional $\exp <-E-S_{j}, j=1:$ size(index,1).

Set cashflow $(\operatorname{index}(j))=\left(E-S_{j}\right), j=1$ :size $($ index, 1$)$.

End if.

Set cashflow $=$ cashflow $e^{(-r \delta t)}$.

End if.

Set American put option $=\operatorname{mean}($ cashflow $)$.

we apply the above LSM algorithm for pricing American put option when the underlying stock price follows the MFH model. The details of LSM Algorithm can be found in [21]. The concept of the put option is related to stopping time process. Indeed, it can be expired at any time until expiration date. Let $\Theta$ be a set of stopping times and $S_{t}$ be a stock price. The price of the American put option is defined as follows

$$
P(\tau, S(\tau))=\sup \left\{\mathbb{E}\left[e^{-r \tau}(E-S(\tau))^{+}\right]\right\}, \tau \in \Theta,
$$

where $S(0)$ is initial stock price.

If $\tau=+\infty$, then the value of the American put option is zero. Here, we investigate the value of the American put option of the MFH model by considering different values of the expiration date and $a, b$ parameter. 


\subsection{Experimental results}

In this section, we present some numerical results for the institution the price of the American put option of the MFH model by using the above LSM algorithm. We first simulate the price of the action, the paths based on the Euler scheme described in subsection 3.2. We use the parameters given in the table 1 for the price of the American put option under the MFH model. By applying the algorithm 1 and 2, we get the following results for differents value of Hurst parameters $H$ such that $H \in\left(\frac{3}{4}, 1\right]$ :

\begin{tabular}{cccc}
\hline$a$ & $b$ & 2 & 3 \\
\hline 0.1 & MFH & 49.7453 & 49.6720 \\
\hline 0.2 & HM & 49.5860 & 49.6969 \\
\hline
\end{tabular}

Table 2: Comparison of American put option using MFH models as function of $S_{0}$ and $V_{0}$ for $\mathbf{H}=\mathbf{0 . 7 6 .}$

\begin{tabular}{cccc}
\hline$a$ & $b$ & 2 & 3 \\
\hline 0.1 & MFH & 49.7970 & 49.6887 \\
\hline 0.2 & HM & 49.6450 & 49.7773 \\
\hline
\end{tabular}

Table 3: Comparison of American put option using MFH models and HM as function of $S_{0}$ and $V_{0}$ for $\mathbf{H}=\mathbf{0 . 7 7}$.

\begin{tabular}{cccc}
\hline$a$ & $b$ & 2 & 3 \\
\hline 0.1 & MFH & 49.7305 & 49.7286 \\
\hline 0.2 & HM & 49.5938 & 49.6614 \\
\hline
\end{tabular}

Table 4: Comparison of American put option using MFH models and HM as function of $S_{0}$ and $V_{0}$ for $\mathbf{H}=\mathbf{0 . 7 8}$.

\begin{tabular}{cccc}
\hline$a$ & $b$ & 2 & 3 \\
\hline 0.1 & MFH & 49.7298 & 49.6620 \\
\hline 0.2 & HM & 49.9172 & 49.7155
\end{tabular}

Table 5: Comparison of American put option using MFH model and HM as function of $S_{0}$ and $V_{0}$ for $\mathbf{H}=\mathbf{0 . 7 9}$

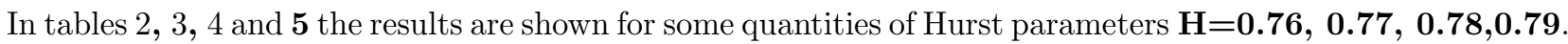
We see that increase in the value of the Hurst parameter leads to a significant increase in the value of the American put option price under MFH.

\begin{tabular}{cccc}
\hline$a$ & $b$ & 2 & 3 \\
\hline 0.1 & MFH & 49.5432 & 49.7045 \\
\hline 0.2 & HM & 49.5249 & 49.7619
\end{tabular}

Table 6: Comparison of American put option using MFH model and HM as function of $S_{0}$ and $V_{0}$ for $\mathbf{H}=\mathbf{0 . 8 0}$. 


\begin{tabular}{cccc}
\hline$a$ & $b$ & 2 & 3 \\
\hline 0.1 & MFH & 49.9421 & 49.7051 \\
\hline 0.2 & HM & 49.6671 & 49.6763 \\
\hline
\end{tabular}

Table 7: Comparison of American put option using MFH model and HM as function of $S_{0}$ and $V_{0}$ for $\mathbf{H}=\mathbf{0 . 8 5}$.

\begin{tabular}{cccc}
\hline$a$ & $b$ & 2 & 3 \\
\hline 0.1 & MFH & 49.8725 & 49.7520 \\
\hline 0.2 & HM & 49.8213 & 49.7047 \\
\hline
\end{tabular}

Table 8: Comparison of American put option using MFH model and HM as function of $S_{0}$ and $V_{0}$ for $\mathbf{H}=\mathbf{0 . 8 7}$.

\begin{tabular}{cccc}
\hline$a$ & $b$ & 2 & 3 \\
\hline 0.1 & MFH & 49.8149 & 49.7072 \\
\hline 0.2 & HM & 49.6484 & 49.7865 \\
\hline
\end{tabular}

Table 9: Comparison of American put option using MFH model and HM as function of $S_{0}$ and $V_{0}$ for $\mathbf{H}=\mathbf{0 . 8 9}$.

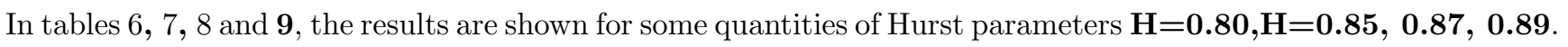
We see that increase in the value of the Hurst parameter leads to a significant increase in the value of the American put option price under $\mathrm{MFH}$.

\begin{tabular}{cccc}
\hline$a$ & $b$ & 2 & 3 \\
\hline 0.1 & MFH & 49.7141 & 49.6956 \\
\hline 0.2 & HM & 49.6721 & 49.6781 \\
\hline
\end{tabular}

Table 10: Comparison of American put option using MFH model and HM as function of $S_{0}$ and $V_{0}$ for $\mathbf{H}=\mathbf{0 . 9 0}$.

\begin{tabular}{cccc}
\hline$a$ & $b$ & 2 & 3 \\
\hline 0.1 & MFH & 49.8439 & 49.7099 \\
\hline 0.2 & HM & 49.9487 & 49.6653 \\
\hline
\end{tabular}

Table 11: Comparison of American put option using MFH model and HM as function of $S_{0}$ and $V_{0}$ for $\mathbf{H}=\mathbf{0 . 9 5}$.

\begin{tabular}{cccc}
\hline$a$ & $b$ & 2 & 3 \\
\hline 0.1 & MFH & 49.7129 & 49.6898 \\
\hline 0.2 & HM & 49.8712 & 49.7194 \\
\hline
\end{tabular}

Table 12: Comparison of American put option using MFH model and HM as function of $S_{0}$ and $V_{0}$ for $\mathbf{H}=\mathbf{0 . 9 7}$.

\begin{tabular}{cccc}
\hline$a$ & $b$ & 2 & 3 \\
\hline 0.1 & MFH & 49.7509 & 49.6964 \\
\hline 0.2 & HM & 49.7540 & 49.7373 \\
\hline
\end{tabular}


Table 13: Comparison of American put option using MFH model and HM as function of $S_{0}$ and $V_{0}$ for $\mathbf{H}=\mathbf{0 . 9 9}$.

In tables 10, 11, 12 and $\mathbf{1 3}$, the results are shown for some quantities of Hurst parameters $\mathbf{H}=\mathbf{0 . 9 0}, \mathbf{H}=\mathbf{0 . 9 5}, \mathbf{0 . 9 7}, \mathbf{0 . 9 9}$. We see that increase in the value of the Hurst parameter leads to a significant increase in the value of the American put option price under MFH.

\section{Conclusion}

In this paper, we have studied the application of LSM algorithm to estimate the value of American put option price of the MFH model that both the stock price and volatility in the model are governed by distinct processes. For this reason, this version of the Heston model that we have proposed in this paper is intuitive and computational efficient. We have proved that our model has a unique solution. Moreover, we have used Euler discretization method which performed the MFH model. Numerical examples showed that the LSM algorithm produces acceptable results which generalized those of the Heston model.

\section{Acknowledgements}

The second author received scholarship for his Doctoral studies from the Non-Governmental Organization "Jean Felicien Gacha's Foundation". And the research work has been done under the research Grant No 17-497RG/MATHS/AF/ACG $-F R 3240297728$ offered by the World Academy of Sciences (TWAS) to the Applied Mathematics to Social Sciences Research Group of the Laboratory of Mathematics-University of Douala-Cameroon. The authors sincerely thanks Jean Felicien Gacha's Foundation and TWAS for their immeasurable help.

\section{References}

[1] S. Albeverio, Z. Brzeźniak, and J-L. Wu. Existence of global solutions and invariant measures for stochastic differential equations driven by poisson type noise with non-lipschitz coefficients. Journal of Mathematical Analysis and Applications, 371(1):309-322, 2010.

[2] E. Alos, J. A. León, D. Nualart, et al. Stochastic stratonovich calculus fbm for fractional brownian motion with hurst parameter less than 1/2. Taiwanese Journal of Mathematics, 5(3):609-632, 2001.

[3] E. Alòs and D. Nualart. Stochastic integration with respect to the fractional brownian motion. Stochastics and Stochastic Reports, 75(3):129-152, 2003.

[4] D. Barbu and G. Bocşan. Approximations to mild solutions of stochastic semilinear equations with non-lipschitz coefficients. Czechoslovak Mathematical Journal, 52(1):87-95, 2002.

[5] M. T. Barlow. One dimensional stochastic differential equations with no strong solution. Journal of the London Mathematical Society, 2(2):335-347, 1982.

[6] J. Barraquand and D. Martineau. Numerical valuation of high dimensional multivariate american securities. Journal of financial and quantitative analysis, 30(3):383-405, 1995. 
[7] F. Biagini, Y. Hu, B. Øksendal, and T. Zhang. Stochastic calculus for fractional Brownian motion and applications. Springer Science \& Business Media, 2008.

[8] M. Broadie, P. Glasserman, and G. Jain. Enhanced monte carlo estimates for american option prices. Journal of Derivatives, 5:25-44, 1997.

[9] P. Carmona, L. Coutin, and booktitle=Annales de l'Institut Henri Poincare (B) Probability and Statistics volume $=39$ number $=1$ pages $=27-68$ year $=2003$ organization $=$ No longer published by Elsevier Montseny, G. Stochastic integration with respect to fractional brownian motion.

[10] P. Cheridito et al. Mixed fractional brownian motion. Bernoulli, 7(6):913-934, 2001.

[11] J. L. da Silva, M. Erraoui, and E. H. Essaky. Mixed stochastic differential equations: Existence and uniqueness result. Journal of Theoretical Probability, 31(2):1119-1141, 2018.

[12] G-F Djang. A modified method of iteration of the picard type in the solution of differential equations. Journal of the Franklin Institute, 246(6):453-457, 1948.

[13] Eric Djeutcha, Didier Alain Njamen Njomen, and Louis-Aimé Fono. Solving arbitrage problem on the financial market under the mixed fractional brownian motion with hurst parameter $\in 1 / 2,3 / 4$. Journal of Mathematics Research, 11(1):76-92, 2019.

[14] T. E. Duncan, Y. Hu, and B. Pasik-Duncan. Stochastic calculus for fractional brownian motion i. theory. SIAM Journal on Control and Optimization, 38(2):582-612, 2000.

[15] S. L. Heston. A closed-form solution for options with stochastic volatility with applications to bond and currency options. The review of financial studies, 6(2):327-343, 1993.

[16] D. J. Higham. An algorithmic introduction to numerical simulation of stochastic differential equations. SIAM review, 43(3):525-546, 2001.

[17] H. E. Hurst. Long-term storage capacity of reservoirs. Trans. Amer. Soc. Civil Eng., 116:770$799,1951$.

[18] A. N. Kolmogorov. Wienersche spiralen und einige andere interessante kurven in hilbertscen raum, cr (doklady). Acad. Sci. URSS (NS), 26:115-118, 1940.

[19] W. E. Leland, M. S. Taqqu, W. Willinger, and D. V. Wilson. On the self-similar nature of ethernet traffic (extended version). IEEE/ACM Transactions on Networking (ToN), 2(1):1$15,1994$.

[20] J. Liu. The law of a stochastic integral with two independent bifractional brownian motions. Communications of the Korean Mathematical Society, 26(4):669-684, 2011.

[21] F. A. Longstaff and E. S. Schwartz. Valuing american options by simulation: a simple leastsquares approach. The review of financial studies, 14(1):113-147, 2001.

[22] B. B. Mandelbrot. The variation of certain speculative prices. In Fractals and scaling in finance, pages 371-418. Springer, 1997.

[23] B. B. Mandelbrot and J. W. Van Ness. Fractional brownian motions, fractional noises and applications. SIAM review, 10(4):422-437, 1968. 
[24] F. Mehrdoust, A. R. Najafi, S. Fallah, and O. Samimi. Mixed fractional heston model and the pricing of american options. Journal of Computational and Applied Mathematics, 330:141-154, 2018.

[25] I. S. Mishura, I. S. Mishura, Y. Mishura, J. S. Mišura, and Û. S. Mišura. Stochastic calculus for fractional Brownian motion and related processes, volume 1929. Springer Science \& Business Media, 2008.

[26] F. Russo and P. Vallois. Forward, backward and symmetric stochastic integration. Probability theory and related fields, 97(3):403-421, 1993.

[27] T. Taniguchi. The existence and uniqueness of energy solutions to local non-lipschitz stochastic evolution equations. Journal of Mathematical Analysis and Applications, 360(1):245-253, 2009.

[28] Y. Xu, B. Pei, and J-L Wu. Stochastic averaging principle for differential equations with non-lipschitz coefficients driven by fractional brownian motion. Stochastics and Dynamics, 17(02):1750013, 2017.

[29] T. Yamada. On a comparison theorem for solutions of stochastic differential equations and its applications. 1973.

[30] T. Yamada et al. On the successive approximation of solutions of stochastic differential equations. Journal of Mathematics of Kyoto University, 21(3):501-515, 1981.

[31] T. Yamada, Shinzo Watanabe, et al. On the uniqueness of solutions of stochastic differential equations. Journal of Mathematics of Kyoto University, 11(1):155-167, 1971. 